\title{
The effects of seasonally adjusting a periodic autoregressive process
}

\author{
Philip Hans Franses \\ Econometric Institute, Erasmus University, Rotterdam P.O. Box 1738 , \\ NL-3000 DR Rotterdam, Netherlands
}

\begin{abstract}
Traditional methods for the analysis of seasonal and nonstationary time series assume that seasonality and a stochastic trend can be separated in some way. However, several macroeconomic time series display patterns which indicate that separation may not be valid. Such patterns occur if seasonal movements change slowly over time and the timing of changes depends on exogenous shocks from e.g., a business cycle. Periodic autoregressive processes with unit roots are suitable for modeling and forecasting such series. Using Monte-Carlo simulations the Census X-11 adjustment and the Box-Jenkins analysis are compared considering the case that the data are generated by periodic processes. It appears, for example, that the intrinsic periodicity is removed only partially, that a test for a unit root is robust, and that the most sensible practical strategy seems to be to start with a general periodic autoregressive model. If necessary, one can then switch to a usual seasonal adjustment procedure if the hypothesis of nonperiodicity cannot be rejected. The quartely real German GNP series is used to illustrate that a periodic model can yield superior modeling and forecasting.
\end{abstract}

\section{Introduction}

Traditional time series methods to analyze seasonally observed variables often assume that seasonality can be removed from the time series, either by using seasonal adjustment filters like the Census X-11 filter or by transforming the series to, e.g., annual growth rates. The main motivations for this practice are that seasonality is considered to be a contamination of the series, and that seasonal fluctuations seem to be reasonably independent of underlying stochastic trends. An economic motivation is that it is often assumed that economic agents abstract from seasonality when forming expectations or plans. 


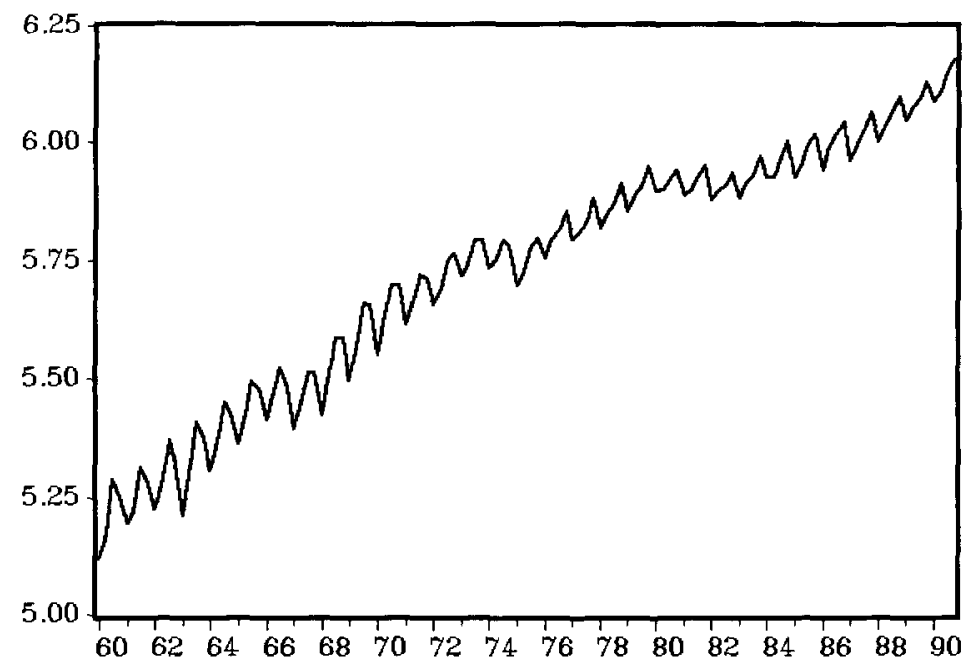

Fig. 1. Vertical axis: the $\log$ of the real German GNP. Horizontal axis: the time in quarters, 1960.1-1990.4.

Recently, there has emerged a growing interest in modeling seasonality in its own right. Beaulieu et al. (1992) found that for the real GNP series in many OECD countries the seasonal variation and the business variation are related. Ghysels (1991) documents for the US composite leading indicator index that business cycle turning points are not equally distributed over the year. Hence, the length of a business cycle may be dependent on the season in which a contraction or expansion starts. In Franses (1992a) it is illustrated with several consumer expectations indices for eight European countries that these show seasonal patterns, suggesting that economic agents do not entirely abstract from seasonal fluctuations. In summary, for some macroeconomic variables it appears that the standard assumptions underlying the traditional analysis may be invalidated.

A typical example of such a macroeconomic time series is the log of the quarterly observed real gross national product (GNP) for Germany, 1960.1-1990.4, as depicted in Fig. 1. This graph shows the characteristics of many quarterly macroeconomic time series, i.e., there is an upward trending pattern and there are marked seasonal fluctuations. Whether the latter fluctuations are constant over time can roughly be observed from the plots in Fig. 2. The quarterly time series is denoted as $y_{t}$, This series can be split up into four annual series, say $Y_{s T}$, containing for each year $T$ the observations per season $s$, where $s=1,2,3,4$. In Fig. 2, the graphs of the $Y_{s T}$ are given. It can be seen that the seasonal pattern seems reasonably constant, albeit that the observations in seasons 3 and 4 change place, and, second, that the differences between the seasons decrease. The latter is more clearly seen from Fig. 3, in which the first differences of $y_{t}$ per season, i.e., $Y_{s T}-Y_{s-1, T}$ for $s=2,3,4$, are displayed. Before 1974 there appear to be distinctive differences between the seasons, although these differences get smaller with additional observations, and after 1975 the differences seem to vanish. Given that the first differenced time series 


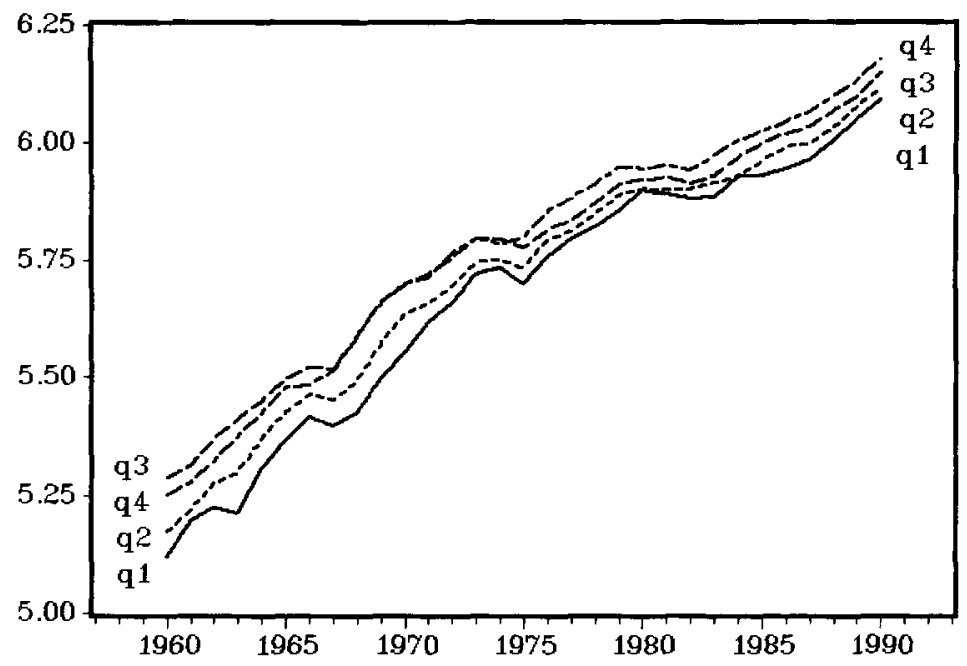

Fig. 2. Vertical axis: the log of real German GNP observed in quarter $s$ with $s=1,2,3,4$ Horizontal axis: the time in years, 1960-1990.

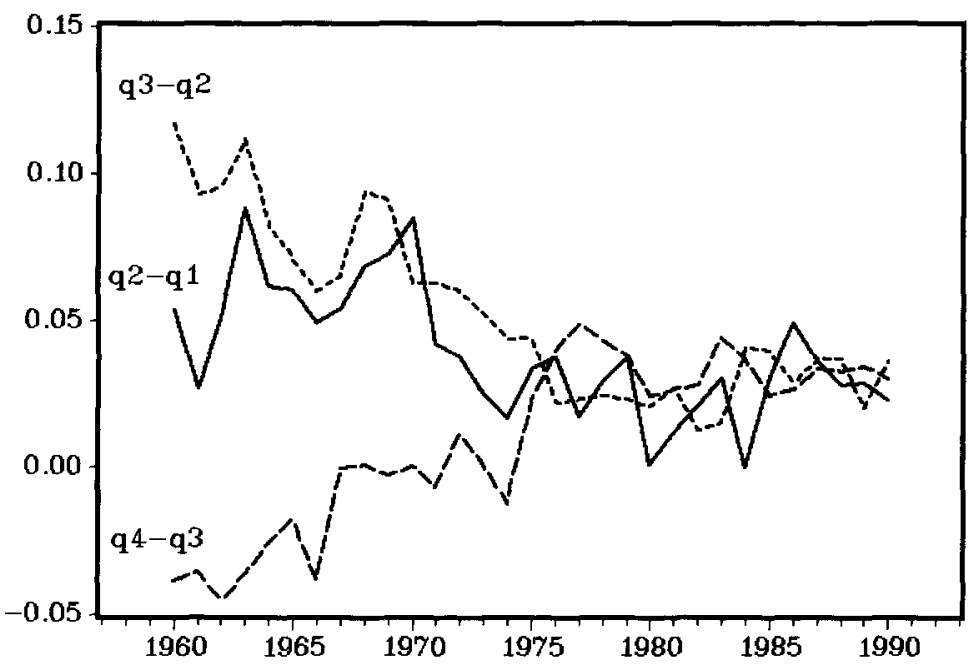

Fig. 3. Vertical axis: the first differences of $y_{t}$ in quarters 2,3 and 4. Horizontal axis: the time in years, 1960-1990.

still displays nonstationary patterns, one may expect that the application of the first differencing filter does not result in a time series without one or more stochastic trends. However, the first-order differenced time series seems stationary after 1975. Hence the application of, e.g., the fourth-order differencing filter to $y_{t}$ to remove possible stochastic trends may introduce unit roots in the moving average polynomial in the second part of the sample. Given that 1975 corresponds to the oil crisis period, it may be the case that the underlying stochastic trend affects the 


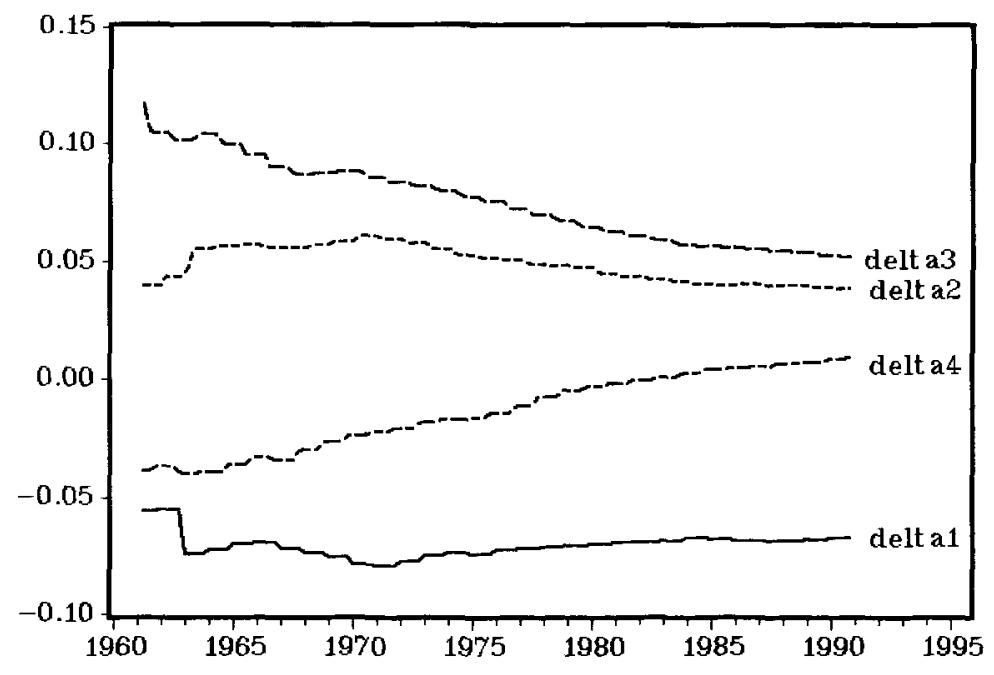

Fig. 4. Vertical axis: recursive OLS estimates of $\delta_{i}$ in the regression $\Delta_{1} y_{t}=\sum_{i=1}^{4} \delta_{i} D_{s t}+v_{t}$, where $D_{s t}$ are seasonal dummy variables Horizontal axis: the time in years.

seasonal pattern. Of course, one cannot exclude the possibility of a deterministic structural change. In summary, an underlying assumption of the separation of trends and seasonal fluctuations, required for a traditional analysis of the German output series may be invalidated. An additional piece of visual evidence for this conjecture is given in Fig. 4. This figure displays the recursive Ordinary Least Squares (OLS) parameter estimates for the four seasonal dummies from a regression of the quarterly growth rates, i.e., $y_{t}-y_{t-1}$, on these variables, see (Barsky and Miron 1989). It is clear from these four graphs that the estimates for the seasonal means are not constant over time, and that a marked change can be noticed somewhere in the beginning of the seventies, see (Canova and Ghysels, forthcoming) for formal tests.

Models that can cope with phenomena as slowly changing seasonal patterns which may be dependent on stochastic trends are periodic models with a single unit root, i.e., time series models which allow the dynamic parameters and the stochastic trend to vary over the season. Indeed, these models assume that each season can be modeled differently. Examples of the empirical use of such periodic time series models are given in (Osborn 1988; Osborn and Smith, 1989; Boswijk and Franses, 1992; Franses, to appear). In (Osborn and Smith 1989; Franses, 1992b; Franses and Romijn, to appear) it is shown that the one-step-ahead forecasting performance can be improved using periodic models with unit roots. In Section 2 of the present paper, a review of some important aspects of periodic autoregressive models with a single unit root is given.

Currently, the use of periodic time series models, with or without unit roots, is not widespread in empirical economics. This means that in cases where periodic models would have been more appropriate, one sticks to the traditional methods to analyze seasonal time series. It is therefore of interest to have an indication of the effects of misspecifying the underlying dynamic periodicity, i.e., of the effects of 
using seasonal correction methods when data are generated from a periodic process. A discussion of the results of several Monte-Carlo exercises is given in Section 3. These exercises consider tests for periodicity and for a unit root, and the identification and testing of nonperiodic models. Thereby, a Monte-Carlo study gives empirical background to some of the theoretical results derived by Tiao and Grupe (1980), Osborn (1991) and Ghysels and Hall (1992). From the experiments it emerges that it seems most appropriate in practice to start an analysis of a seasonally observed time series with a test for periodically varying dynamics. Further, unit root testing in a periodic model does not seem to be much affected when a linear Census X-11 correction filter is used. Moreover, the intrinsic periodicity is often removed only to a small extent. Section 4 is dedicated to a profound analysis of the German data discussed above. The final section discusses some implications and extensions of the empirical results in this paper.

\section{Periodic autoregressive processes}

A general form of a periodic autoregressive process of order $p, \operatorname{PAR}(p)$, for a possibly trended quarterly time series $y_{t}$ is

$$
y_{t}=\sum_{s=1}^{4}\left[\mu_{s} D_{s t}+\delta_{s} D_{s t} T_{t}\right]+\sum_{i=1}^{p} \sum_{s=1}^{4} D_{s t} \phi_{i s} y_{t-i}+\varepsilon_{t},
$$

where $D_{s t}$ are four seasonal dummies, $\mu_{s}, \delta_{s}$ and $\phi_{i s}$ are periodically varying parameters, $T_{t}$ is a deterministic trend variable, and $\varepsilon_{t}$ denotes a standard white noise process. Of course, not all $\phi_{i s}$ have to be nonzero, and hence $p$ is the maximum of the orders $p_{s}$ per season. Further, $\varepsilon_{t}$ can be replaced by $\varepsilon_{s t}$ to allow for seasonally varying error variances. The model in (1) can be estimated using ordinary least squares.

Note that the seasonal variation in $\mu_{s}$ and $\delta_{s}$ is not necessarily caused by a nonconstant underlying trend and mean. This is illustrated with the simple PAR(1) model without a linear trend, or

$$
y_{t}-\mu=\gamma_{s}\left(y_{t-1}-\mu\right)+\varepsilon_{t},
$$

which can be rewritten as

$$
y_{t}=\gamma_{s} y_{t-1}+\mu_{s}+\varepsilon_{t},
$$

with $\mu_{s}=\left(1-\gamma_{s}\right) \mu$. Hence, although the mean of the series $y_{t}$ is constant over all seasons, a regression model for $y_{t}$ should include four seasonal dummies. Model (1) is of course not expected to be valid for any seasonally observed series. One may want to test whether indeed the $\phi_{i s}$ are periodic. In (Boswijk and Franses, 1992) it is shown that a standard F-test for the null hypothesis $\mathrm{H}_{0}: \phi_{i s}=\phi_{i}$ for $s=1,2,3,4$ can be performed. The distribution of this test statistic is not affected by the stationarity properties of $y_{t}$. Moreover, note from (2) that seasonality and dynamics cannot be separated in a PAR model. This already suggests that a linear filtering of the $y_{t}$ series in (2) will not likely remove all periodicity. In Section 3 below, this conjecture is analyzed in detail. 
An alternative representation of model (1), which facilitates an analysis of its stationarity properties, (see Tiao and Grupe, 1980; Osborn, 1991 and Franses, 1991), inter alia, is given by stacking the annual series with the observations per season, $Y_{\mathrm{s} T}$, in a $(4 \times 1)$ vector. Note that the index $T=1, \ldots, N$, is used for annual data and that index $t=1, \ldots, n$ where $n / N=4$ is used for quarterly data. The model (1) can be written in vector notation as

$$
A_{0}\left[\begin{array}{l}
Y_{1 T} \\
Y_{2 T} \\
Y_{3 T} \\
Y_{4 T}
\end{array}\right]=\left[\begin{array}{l}
\mu_{1} \\
\mu_{2} \\
\mu_{3} \\
\mu_{4}
\end{array}\right]+\left[\begin{array}{l}
\delta_{1} \\
\delta_{2} \\
\delta_{3} \\
\delta_{4}
\end{array}\right] T_{T}+A_{1}\left[\begin{array}{l}
Y_{1 T-1} \\
Y_{2 T-1} \\
Y_{3 T-1} \\
Y_{4 T-1}
\end{array}\right]+A_{2}\left[\begin{array}{l}
Y_{1 T-2} \\
Y_{2 T-2} \\
Y_{3 T-2} \\
Y_{4 T-2}
\end{array}\right]+\cdots+\omega_{T},
$$

where the $A_{i}, i=0,1, \ldots$, are $(4 \times 4)$ parameter matrices containing the various $\phi_{i s}$, $T_{T}$ corresponds to the $T_{t}$ variable in (1), and $\omega_{T}$ is a vector white noise process containing the stacked $\varepsilon_{t}$ 's. The stationarity condition for the process $Y_{T}$, and hence for the univariate process $y_{t}$, is that the roots of the equation

$$
\left|A_{0}-A_{1} z-A_{2} z^{2}-\cdots\right|=0,
$$

are outside the unit circle. When $z=1$ is the only unity solution of (5), while all other $|z|>1$, the process is called periodically integrated of order $1, \operatorname{PI}(1)$, see (Osborn, 1988) and (Boswijk and Franses, 1992). Periodic integration usually involves a nonlinear parameter restriction on the autoregressive parameters in (1). An example is the simple PAR(1) process

$$
y_{t}=\phi_{1 s} y_{t-1}+\varepsilon_{t},
$$

for which the $A_{0}$ and $A_{1}$ are given by

$$
A_{0}=\left[\begin{array}{cccc}
1 & 0 & 0 & 0 \\
-\phi_{12} & 1 & 0 & 0 \\
0 & -\phi_{13} & 1 & 0 \\
0 & 0 & -\phi_{14} & 1
\end{array}\right], \quad A_{1}=\left[\begin{array}{cccc}
0 & 0 & 0 & \phi_{11} \\
0 & 0 & 0 & 0 \\
0 & 0 & 0 & 0 \\
0 & 0 & 0 & 0
\end{array}\right],
$$

and the characteristic equation is

$$
\left|A_{0}-A_{1} z\right|=\left(1-\phi_{11} \phi_{12} \phi_{13} \phi_{14} z\right)=0 .
$$

The process $y_{t}$ in (6) is periodically stationary if $\left|\phi_{11} \phi_{12} \phi_{13} \phi_{14}\right|<1$ and the process is $\mathrm{PI}(1)$ when $\phi_{11} \phi_{12} \phi_{13} \phi_{14}=1$. The appropriate differencing filter for a $\mathrm{PI}(1)$ series in case of $(6)$ is $\left(1-\phi_{1 s} B\right)$ with $\phi_{11} \phi_{12} \phi_{13} \phi_{14}=1$, where $B$ is the usual backward shift operator defined by $B^{k} x_{t}=x_{t-k}$.

In (Boswijk and Franses, 1992) a test for periodic integration is proposed for general PAR models as in (1). This likelihood ratio test statistic BF is given by

$$
\mathrm{BF}=[\operatorname{sign}(g(\hat{\phi})-1)]\left[n \log \left(\mathrm{RSS}_{0} / \mathrm{RSS}_{1}\right)\right]^{1 / 2},
$$


where $\mathrm{RSS}_{0}$ and $\mathrm{RSS}_{1}$ are the estimated residual sums of squares of the restricted and unrestricted models, log denotes natural logarithm, and $g(\hat{\phi})$ is a function of the parameters. For model (6), $g(\phi)$ equals $\phi_{11} \phi_{12} \phi_{13} \phi_{14}$. The asymptotic distribution of the BF statistic is tabulated in (Fuller, 1976, Table 8.5.2). In (Boswijk and Franses, 1992) two alternative tests are proposed for integration in a PAR(1) model. From simulation experiments it emerges that the BF test in (9) has the best empirical performance.

An additional advantage of the BF test is that it can be used in extended PAR models as well. For example, the periodic AR(2) process

$$
y_{t}=\phi_{1 s} y_{t-1}+\phi_{2 s} y_{t-2}+\varepsilon_{t},
$$

with its multivariate representation

$$
A_{0} Y_{T}=A_{1} Y_{T-1}+\omega_{T},
$$

with

$$
A_{0}=\left[\begin{array}{cccc}
1 & 0 & 0 & 0 \\
-\phi_{12} & 1 & 0 & 0 \\
-\phi_{23} & -\phi_{13} & 1 & 0 \\
0 & -\phi_{24} & -\phi_{14} & 1
\end{array}\right] \quad A_{1}=\left[\begin{array}{cccc}
0 & 0 & \phi_{21} & \phi_{11} \\
0 & 0 & 0 & \phi_{22} \\
0 & 0 & 0 & 0 \\
0 & 0 & 0 & 0
\end{array}\right],
$$

is stationary if the roots of the characteristic equation

$$
\begin{aligned}
\left|A_{0}-A_{1} z\right|=1-[ & \phi_{22} \phi_{13} \phi_{14}+\phi_{22} \phi_{24}+\phi_{21} \phi_{12} \phi_{13}+\phi_{21} \phi_{23} \\
& \left.+\phi_{11} \phi_{12} \phi_{13} \phi_{14}+\phi_{11} \phi_{12} \phi_{24}+\phi_{11} \phi_{14} \phi_{23}\right] z \\
& +\phi_{21} \phi_{22} \phi_{23} \phi_{24} z^{2}=0,
\end{aligned}
$$

are outside the unit circle. If $z$ is equal to 1 , the expression in (12) can be expressed as a nonlinear parameter restriction. Standard nonlinear least squares routines can then be used to calculate the BF test statistic in (9). An alternative form of (10) is

$$
y_{t}-\alpha_{s} y_{t-1}=\beta_{s}\left(y_{t-1}-\alpha_{s-1} y_{t-2}\right)+\varepsilon_{t},
$$

where $\alpha_{s}$ and $\beta_{s}$ are functions of the $\phi_{1 s}$ and $\phi_{2 s}$, with $\alpha_{-k}=\alpha_{s-k}$ for $k=0,1, \ldots$ Rewriting (13) in stacked form, it can be derived that the characteristic equation $\left|A_{0}-A_{1} z\right|=0$ becomes $\left(1-\alpha_{1} \alpha_{2} \alpha_{3} \alpha_{4} z\right)\left(1-\beta_{1} \beta_{2} \beta_{3} \beta_{4} z\right)=0$. Hence, the differencing filter for a $\mathrm{PI}(1)$ process when the autoregressive order is 2 , or higher, is $\left(1-\alpha_{s} B\right)$ with the restriction that $\alpha_{1} \alpha_{2} \alpha_{3} \alpha_{4}=1$. Note that $\left(1-\alpha_{s} B\right) \approx(1-B)$, since the $(1-B)$ filter assumes that all $\alpha_{s}$ are equal to unity. It is also clear that the $\left(1-\alpha_{s} B\right)$ filter allows for the stochastic trend to vary with the seasons. Furthermore, if $\alpha_{s} \neq 1, Y_{T}$ can converge or diverge, depending on the underlying stochastic trend. This property ensures that a PI(1) process can be useful to describe some macroeconomic time series. Finally, it should be mentioned that not all $\beta_{s}$ in (13) have to be nonzero, i.e., the lag lengths in a $\operatorname{PAR}(p)$ process do not have to be equal. A sensible model selection strategy seems to be to estimate in a first step the parameters of a general $\operatorname{PAR}(p)$ process, then in a second step to check whether 
a restriction like $\alpha_{1} \alpha_{2} \alpha_{3} \alpha_{4}=1$ holds, and finally, to test whether some $\beta_{s}$ can be set equal to zero. One can use standard $t$ type tests since the $\left(1-\alpha_{s} B\right) y_{t}$ series under the restriction $\alpha_{1} \alpha_{2} \alpha_{3} \alpha_{4}=1$ is a (periodically) stationary time series.

If a PAR(1) process is periodically integrated, or if $\phi_{11} \phi_{21} \phi_{31} \phi_{41}=1$ in (6), then there are three cointegration relations between the $Y_{s T}$ series. This is easily seen by rewriting

$$
Y_{T-1} Y_{T-1}=\left(A_{0}^{-1} A_{1}-I\right) Y_{T-1}+v_{T},
$$

where $v_{t}=A_{0}^{-1} w_{T}$, and with

$$
\left(A_{0}^{-1} A_{1}-I\right)=\left[\begin{array}{cccc}
-1 & 0 & 0 & \phi_{11} \\
0 & -1 & 0 & \phi_{11} \phi_{12} \\
0 & 0 & -1 & \phi_{11} \phi_{12} \phi_{13} \\
0 & 0 & 0 & \phi_{11} \phi_{12} \phi_{13} \phi_{14}-1
\end{array}\right] \text {. }
$$

Evidently, if $\prod_{s=1}^{4} \phi_{1 s}=1$ the matrix $A_{0}^{-1} A_{1}-I$ has rank 3. For out-of-sample forecasting this means that the forecasts for the annual series are tied together and, hence, that a forecasting improvement can be expected, see (Engle and Yoo, 1987).

Note that $Y_{T}-Y_{T-1}$ corresponds to the fourth-order differencing filter for quarterly series. Hence, the use of this filter would imply overdifferencing in case the $y_{t}$ series is periodically integrated. Since the $\Delta_{4}$ filter for $y_{t}$ is only one way of deseasonalizing a time series where $\Delta_{k} x_{t}=x_{t}-x_{t-k}$, it is of interest to investigate the effects of seasonal adjustment of a series generated by a PAR process as in (1). The next section reports on a Monte-Carlo study of such effects.

\section{Some Monte-Carlo Results}

Consider the quarterly time series $y_{t}$ generated by a periodic AR process of order 1 , or $y_{t}=\phi_{s} y_{t-1}+\varepsilon_{t}$, with $s=1,2,3,4$, and $\varepsilon_{t}$ as a Gaussian white noise process. In Table 1, the six data generating processes (DGP) are given which will be used in

Table 1

\begin{tabular}{|c|c|c|c|c|c|}
\hline DGP & $\phi_{1}$ & $\phi_{2}$ & $\phi_{3}$ & $\phi_{4}$ & $\phi_{1} \phi_{2} \phi_{3} \phi_{4}$ \\
\hline & \multicolumn{5}{|c|}{ Periodically stationary processes } \\
\hline i & 0.4 & 1.2 & 0.8 & 1.6 & 0.6144 \\
\hline ii & 0.6 & 0.8 & 1.0 & 1.2 & 0.5760 \\
\hline iii & 1.3 & 0.3 & 1.5 & 1.3 & 0.7605 \\
\hline \multirow[t]{2}{*}{ iv } & 0.7 & 0.95 & 1.2 & 1.0 & 0.7980 \\
\hline & \multicolumn{5}{|c|}{ Periodically integrated processes } \\
\hline $\mathrm{v}$ & 0.5 & 0.9 & 1.5 & $1 / \phi_{1} \phi_{2} \phi_{3}$ & 1.0000 \\
\hline vi & 1.1 & 0.91 & 1.05 & $1 / \phi_{1} \phi_{2} \phi_{3}$ & 1.0000 \\
\hline
\end{tabular}

Six data generating processes: $y_{t}=\phi_{s} y_{t-1}+\varepsilon_{t}, \varepsilon_{t} \sim N(0,1) E\left(\varepsilon_{t}, \varepsilon_{t-k}\right) \neq 0$ for all $k \neq 0$ 
all forthcoming simulations. The DGPs (i) through (iv) are periodically stationary, while the DGPs (v) and (vi) are periodically integrated. The first four DGPs are included to enable a comparison of the cases with and without a unit root. All Monte-Carlo simulations below are based on 100 effective observations with 1000 replications in each case. The Gauss 386 programming language is used. The sample size of 100 corresponds to the number of quarterly observations which is often encountered in pratice. Unless indicated, the significance levels are set equal to $5 \%$ in all simulations.

When a researcher is not aware of the underlying data generating process, and proceeds along the standard Box and Jenkins methodology, he will consider the (partial) autocorrelation functions of $y_{t}, \Delta_{1} y_{t}$ (after regression on seasonal dummies to remove possible deterministic seasonality), and $\Delta_{4} y_{t}$. He tries to find out whether these functions give an indication of an appropriate model order. The first three panels of Tables 2 and 3 display the estimated autocorrelations and partial

Table 2

Estimated autocorrelations of $y_{t}, \Delta_{1} y_{t}, \Delta_{4} y_{t}$ and $\tilde{y}_{t}$. DGP is a PAR(1) process: $y_{t}=\phi_{s} y_{t-1}+\varepsilon_{t}$, $\varepsilon_{t} \sim N(0,1)$. The number of observations is 100 . The number of replications is 1000 . The figures in the cells are average estimated autocorrelations

\begin{tabular}{|c|c|c|c|c|c|c|c|}
\hline \multirow[b]{2}{*}{ Series } & \multirow[b]{2}{*}{ Lag } & \multicolumn{3}{|c|}{ Periodically stationary DGP } & \multicolumn{3}{|c|}{ Periodically integrated DGP } \\
\hline & & $\mathrm{i}$ & ii & iii & iv & $\mathrm{v}$ & vi \\
\hline \multirow[t]{12}{*}{ (1) $y_{t}$} & 1 & 0.73 & 0.80 & 0.74 & 0.87 & 0.59 & 0.91 \\
\hline & 2 & 0.65 & 0.64 & 0.60 & 0.75 & 0.33 & 0.86 \\
\hline & 3 & 0.50 & 0.53 & 0.56 & 0.68 & 0.51 & 0.79 \\
\hline & 4 & 0.49 & 0.45 & 0.62 & 0.63 & 0.81 & 0.75 \\
\hline & 5 & 0.34 & 0.35 & 0.44 & 0.54 & 0.44 & 0.68 \\
\hline & 6 & 0.29 & 0.27 & 0.34 & 0.46 & 0.20 & 0.64 \\
\hline & 7 & 0.21 & 0.21 & 0.31 & 0.40 & 0.37 & 0.57 \\
\hline & 8 & 0.21 & 0.17 & 0.36 & 0.36 & 0.66 & 0.54 \\
\hline & 9 & 0.13 & 0.12 & 0.24 & 0.30 & 0.32 & 0.48 \\
\hline & 10 & 0.10 & 0.08 & 0.17 & 0.24 & 0.10 & 0.45 \\
\hline & 11 & 0.06 & 0.05 & 0.15 & 0.20 & 0.26 & 0.40 \\
\hline & 12 & 0.06 & 0.03 & 0.19 & 0.18 & 0.53 & 0.38 \\
\hline \multirow[t]{12}{*}{ (2) $\Delta_{1} y_{t}$} & 1 & -0.31 & -0.07 & -0.19 & -0.01 & -0.11 & -0.07 \\
\hline & 2 & 0.12 & -0.10 & -0.15 & -0.14 & -0.42 & -0.06 \\
\hline & 3 & -0.22 & -0.08 & -0.20 & -0.04 & -0.13 & -0.07 \\
\hline & 4 & 0.19 & 0.00 & 0.37 & 0.05 & 0.59 & 0.03 \\
\hline & 5 & -0.16 & -0.03 & -0.12 & -0.00 & -0.09 & -0.06 \\
\hline & 6 & 0.06 & -0.04 & -0.09 & -0.10 & -0.33 & 0.06 \\
\hline & 7 & -0.10 & -0.04 & -0.12 & -0.02 & -0.11 & -0.06 \\
\hline & 8 & 0.07 & -0.02 & 0.20 & 0.01 & 0.46 & 0.02 \\
\hline & 9 & -0.07 & -0.02 & -0.06 & 0.00 & -0.06 & -0.04 \\
\hline & 10 & 0.02 & -0.02 & -0.05 & -0.05 & -0.25 & 0.04 \\
\hline & 11 & -0.04 & -0.01 & -0.07 & -0.02 & -0.08 & -0.04 \\
\hline & 12 & 0.01 & -0.03 & 0.08 & -0.00 & -0.33 & 0.01 \\
\hline
\end{tabular}


Table 2. (Continued)

\begin{tabular}{|c|c|c|c|c|c|c|c|}
\hline Series & Lag & $\mathrm{i}$ & ii & iii & iv & $\mathrm{v}$ & vi \\
\hline \multirow[t]{12}{*}{ (3) $\Delta_{4} y_{t}$} & 1 & 0.61 & 0.65 & 0.65 & 0.70 & 0.67 & 0.72 \\
\hline & 2 & 0.36 & 0.34 & 0.34 & 0.41 & 0.37 & 0.46 \\
\hline & 3 & 0.08 & 0.06 & 0.08 & 0.15 & 0.15 & 0.20 \\
\hline & 4 & -0.18 & -0.22 & -0.12 & -0.11 & -0.04 & -0.05 \\
\hline & 5 & -0.14 & -0.17 & -0.10 & -0.09 & -0.03 & -0.04 \\
\hline & 6 & -0.13 & -0.14 & -0.08 & -0.08 & -0.03 & -0.04 \\
\hline & 7 & -0.11 & -0.12 & -0.08 & -0.08 & -0.04 & -0.04 \\
\hline & 8 & -0.11 & -0.11 & -0.08 & -0.08 & -0.04 & -0.03 \\
\hline & 9 & -0.09 & -0.10 & -0.06 & -0.07 & -0.04 & -0.03 \\
\hline & 10 & -0.08 & -0.08 & -0.06 & -0.07 & -0.04 & -0.04 \\
\hline & 11 & -0.07 & -0.07 & -0.06 & -0.06 & -0.03 & -0.04 \\
\hline & 12 & -0.07 & -0.06 & -0.06 & -0.06 & -0.03 & -0.04 \\
\hline \multirow[t]{12}{*}{ (4) $\tilde{y}_{t}$} & 1 & 0.82 & 0.84 & 0.87 & 0.90 & 0.92 & 0.94 \\
\hline & 2 & 0.70 & 0.69 & 0.75 & 0.80 & 0.84 & 0.88 \\
\hline & 3 & 0.57 & 0.56 & 0.65 & 0.70 & 0.78 & 0.81 \\
\hline & 4 & 0.45 & 0.43 & 0.57 & 0.61 & 0.72 & 0.74 \\
\hline & 5 & 0.39 & 0.37 & 0.51 & 0.55 & 0.68 & 0.70 \\
\hline & 6 & 0.32 & 0.30 & 0.44 & 0.48 & 0.62 & 0.65 \\
\hline & 7 & 0.25 & 0.23 & 0.37 & 0.41 & 0.57 & 0.59 \\
\hline & 8 & 0.17 & 0.15 & 0.30 & 0.34 & 0.51 & 0.54 \\
\hline & 9 & 0.15 & 0.13 & 0.27 & 0.30 & 0.48 & 0.50 \\
\hline & 10 & 0.11 & 0.09 & 0.23 & 0.26 & 0.44 & 0.46 \\
\hline & 11 & 0.08 & 0.05 & 0.18 & 0.21 & 0.39 & 0.42 \\
\hline & 12 & 0.03 & 0.02 & 0.13 & 0.17 & 0.35 & 0.38 \\
\hline
\end{tabular}

autocorrelations for the six DGPs, respectively. It can be observed from Table 2 that the autocorrelations of $y_{t}$ die out only slowly, and that its fourth autocorrelation coefficient is relatively large. Hence, there is a sign of nonstationarity in the $y_{t}$ series. For $\Delta_{1} y_{t}$ one could be inclined to fit low-order autoregressions. The third panel of Table 2 indicates that the $\Delta_{4} y_{t}$ series displays a AR(1) type of pattern, with a parameter possibly not too close to unity. Hence, a $\Delta_{1} \Delta_{4}$ filter does not seem to be appropriate in most cases. Note that the autocorrelations in the first three panels of Table 2 show many similarities, i.e., whether the DGPs have a unit root or not.

The estimated partial autocorrelations reported in the first three panels of Table 3 indicate that (nonperiodic) autoregressive models seem useful to describe the time series, although the autoregressive orders vary over the different DGPs. For example, an $\operatorname{AR}(1,2)$ process emerges for $y_{t}$ generated by DGP (i), while an $\operatorname{AR}(1,3,4,5)$ results for the $y_{t}$ series generated by $\operatorname{DGP}(v)$, where $\operatorname{AR}\left(p_{1}, p_{2}, \ldots\right)$ denotes an autoregressive model with lags at $p_{1}, p_{2}, \ldots$ In Table 4 , the orders of the tentatively identified nonperiodic models for $y_{t}, \Delta_{1} y_{t}$ and $\Delta_{4} y_{t}$ are given. These models will be used in the subsequent Monte-Carlo studies. Summarizing, reasonably low-order AR models can be identified when PAR(1) processes are the underlying DGPs. 
The transformation $\Delta_{4}$ is used to remove the seasonality of a time series. Alternatively, once can transform the $y_{t}$ into a seasonally adjusted series, to be denoted as $\tilde{y}_{t}$ using a linearized Census X-11 fitler. For quarterly data the filter takes the form

$$
\tilde{y}_{t}=\left[\sum_{i=1}^{-28} a_{i} B^{i}+a_{0}+\sum_{j=1}^{+28} a_{j} B^{j}\right] y_{t}
$$

where the $a_{i}$ are given in (Laroque, 1977). Assuming a PAR(1) process as the DGP, the estimated autocorrelations of $\tilde{y}_{t}$ are displayed in the bottom part of Table 2 . These autocorrelations die out only slowly, even when $\prod_{i=1}^{4} \phi_{1 i}$ is only 0.6 as in the case of DGPs (i) and (ii). The partial autocorrelations in the fourth panel Table 3 for $\tilde{y}_{t}$ suggest that a nonperiodic AR(1) model would be tentatively identified. Note, that the autocorrelations are higher for the $\tilde{y}_{t}$ series than for the $y_{t}$ series. In

Table 3

Estimated partial autocorrelations of $y_{t}, \Delta_{1} y_{t}, \Delta_{4} y_{t}$ and $\tilde{y}_{t}$. DGP is a PAR(1) process: $y_{t}=\phi_{s} y_{t-1}+\varepsilon_{t}$, $\varepsilon_{t} \sim \mathrm{N}(0,1)$. The number of observations is 100 . The number of replications is 1000 . The figures in the cells are average estimated partial autocorrelations coefficients

\begin{tabular}{|c|c|c|c|c|c|c|c|}
\hline \multirow[b]{2}{*}{ Series } & \multirow[b]{2}{*}{ Lag } & \multicolumn{3}{|c|}{ Periodically stationary DGP } & \multicolumn{3}{|c|}{ Periodically integrated DGP } \\
\hline & & $\mathrm{i}$ & ii & iii & iv & $\mathrm{v}$ & vi \\
\hline \multirow[t]{12}{*}{$y_{t}$} & 1 & 0.75 & 0.82 & 0.75 & 0.88 & 0.73 & 0.94 \\
\hline & 2 & 0.25 & -0.02 & 0.11 & -0.04 & -0.00 & 0.14 \\
\hline & 3 & -0.10 & 0.04 & 0.16 & 0.15 & 0.51 & -0.12 \\
\hline & 4 & 0.17 & 0.03 & 0.35 & 0.00 & 0.60 & 0.10 \\
\hline & 5 & -0.19 & -0.09 & -0.38 & -0.14 & -0.60 & -0.13 \\
\hline & 6 & -0.01 & -0.01 & 0.02 & -0.03 & 0.09 & 0.06 \\
\hline & 7 & -0.00 & 0.00 & 0.03 & 0.07 & 0.03 & -0.07 \\
\hline & 8 & 0.01 & 0.00 & 0.02 & -0.01 & 0.09 & 0.04 \\
\hline & 9 & -0.13 & -0.05 & -0.09 & -0.08 & -0.18 & -0.09 \\
\hline & 10 & -0.05 & -0.02 & -0.01 & -0.03 & 0.04 & 0.03 \\
\hline & 11 & -0.02 & -0.01 & -0.00 & 0.02 & -0.02 & -0.05 \\
\hline & 12 & -0.01 & -0.02 & -0.01 & -0.02 & 0.01 & 0.02 \\
\hline \multirow[t]{12}{*}{$\Delta_{1} y_{t}$} & 1 & -0.31 & -0.07 & -0.19 & -0.01 & -0.11 & -0.07 \\
\hline & 2 & 0.02 & -0.11 & -0.20 & -0.15 & -0.45 & 0.05 \\
\hline & 3 & -0.20 & -0.09 & -0.32 & -0.04 & -0.37 & -0.05 \\
\hline & 4 & 0.07 & -0.03 & 0.25 & 0.02 & 0.42 & 0.01 \\
\hline & 5 & -0.07 & -0.05 & -0.07 & -0.01 & -0.10 & -0.04 \\
\hline & 6 & -0.07 & -0.07 & -0.07 & -0.09 & -0.05 & 0.03 \\
\hline & 7 & -0.06 & -0.06 & -0.06 & -0.02 & -0.08 & -0.04 \\
\hline & 8 & -0.05 & -0.06 & -0.01 & -0.03 & 0.07 & -0.01 \\
\hline & 9 & -0.04 & -0.04 & -0.03 & -0.01 & -0.05 & -0.03 \\
\hline & 10 & -0.05 & -0.05 & -0.03 & -0.05 & 0.00 & -0.01 \\
\hline & 11 & -0.03 & -0.03 & -0.02 & -0.02 & -0.02 & -0.03 \\
\hline & 12 & -0.07 & -0.07 & -0.07 & -0.05 & -0.03 & -0.03 \\
\hline
\end{tabular}


Table 3. (Continued)

\begin{tabular}{|c|c|c|c|c|c|c|c|}
\hline Series & Lag & $\mathrm{i}$ & ii & iii & iv & $\mathrm{v}$ & vi \\
\hline \multirow[t]{12}{*}{$\Delta_{4} y_{t}$} & 1 & 0.62 & 0.67 & 0.65 & 0.71 & 0.69 & 0.73 \\
\hline & 2 & -0.04 & -0.18 & -0.14 & -0.18 & -0.17 & -0.15 \\
\hline & 3 & -0.22 & -0.19 & -0.16 & -0.17 & -0.07 & -0.17 \\
\hline & 4 & -0.26 & -0.26 & -0.16 & -0.23 & -0.15 & -0.22 \\
\hline & 5 & 0.23 & 0.29 & 0.19 & 0.32 & 0.18 & 0.34 \\
\hline & 6 & -0.04 & -0.12 & -0.09 & -0.12 & -0.09 & -0.10 \\
\hline & 7 & -0.14 & -0.12 & -0.10 & -0.09 & -0.02 & -0.11 \\
\hline & 8 & -0.17 & -0.16 & -0.10 & -0.13 & -0.06 & -0.12 \\
\hline & 9 & 0.14 & 0.17 & 0.10 & 0.19 & 0.05 & 0.22 \\
\hline & 10 & -0.04 & -0.10 & -0.06 & -0.11 & -0.05 & -0.07 \\
\hline & 11 & -0.10 & -0.09 & -0.06 & -0.05 & -0.01 & -0.09 \\
\hline & 12 & -0.13 & -0.12 & -0.07 & -0.10 & -0.03 & -0.09 \\
\hline \multirow[t]{12}{*}{$\tilde{y}_{t}$} & 1 & 0.84 & 0.85 & 0.88 & 0.91 & 0.95 & 0.96 \\
\hline & 2 & 0.08 & -0.08 & -0.03 & -0.10 & -0.07 & -0.09 \\
\hline & 3 & -0.10 & -0.04 & 0.01 & -0.03 & 0.10 & -0.07 \\
\hline & 4 & -0.07 & -0.09 & 0.02 & -0.09 & -0.03 & -0.09 \\
\hline & 5 & 0.14 & 0.19 & 0.07 & 0.18 & 0.05 & 0.20 \\
\hline & 6 & -0.07 & -0.11 & -0.11 & -0.13 & -0.13 & -0.13 \\
\hline & 7 & -0.07 & -0.06 & -0.05 & -0.04 & -0.02 & -0.07 \\
\hline & 8 & -0.12 & -0.09 & -0.06 & -0.08 & -0.05 & -0.08 \\
\hline & 9 & 0.20 & 0.20 & 0.17 & 0.19 & 0.14 & 0.21 \\
\hline & 10 & -0.09 & -0.13 & -0.13 & -0.16 & -0.14 & -0.14 \\
\hline & 11 & -0.07 & -0.06 & -0.05 & -0.04 & -0.03 & -0.07 \\
\hline & 12 & -0.10 & -0.08 & -0.05 & -0.07 & -0.04 & -0.08 \\
\hline
\end{tabular}

summary, one concludes that a nonperiodic AR model can be used for $y_{t}, \Delta_{1} y_{t}$, $\Delta_{4} y_{t}$, as well as for $\tilde{y}_{t}$ series if a PR(1) process is the DGP.

The parameter estimates of a nonperiodic AR(1) model based on $\tilde{y}_{t}$ are displayed in the first row of Table 5. These parameter estimates must be an average of the parameters in the DGP, see (Tiao and Grupe, 1980). Suppose, one would like to specify a periodic autoregressive process for an adjusted series $\tilde{y}_{t}$. The relevant parameter estimates are given in the second part of Table 5. At first sight, it seems that the values of the estimated parameters still differ per season. This is most clearly seen for the first three DGPs. For example, the PAR(1) parameters for (i) are $0.4,1.2,0.8$ and 1.6, while the estimated parameters for the PAR(1) model for $\tilde{y}_{t}$ are $0.566,1.013,0.872$ and 1.219 , respectively. Additionally, it can be seen that the individual values are closer to unity. The bottom row of Table 5 gives the result of tests for the periodicity of the autoregressive parameter values in a PAR(1) process for $\tilde{y}_{t}$. Evidently, in some cases the rejection frequency is very high while for others it is zero. This indicates that one may find the $(1-B)$ filter to be adequate in cases of PAR(1) where the $\phi_{s} \approx 1$. A further result which is worthwhile noting: the product $\prod_{i=1}^{4} \hat{\phi}_{i}$ of the estimated parameters $\hat{\phi}_{i}$ is almost equal to the product of the 
Table 4

Autoregressive (AR) models identified from the (partial) autocorrelations functions of Tables 2 and 3. The cells indicate the lags to be included in the corresponding AR model ${ }^{1}$

\begin{tabular}{lllllll} 
& \multicolumn{1}{c}{ DGP } & & & & & \\
\cline { 2 - 7 } Series & i & ii & iii & iv & v & vi \\
\hline$y_{t}$ & 1,2 & 1 & $1,4,5$ & 1 & $1,3,4,5$ & 1 \\
$\Delta_{1} y_{t}$ & 1,3 & 0 & $1,2,3,4$ & 0 & $1,2,3,4$ & 0 \\
$\Delta_{4} y_{t}$ & $1,3,4,5$ & $1,2,3,4,5$ & $1,4,5$ & $1,4,5$ & 1 & $1,4,5$ \\
$\tilde{y}_{t}$ & 1 & 1 & 1 & 1 & 1 & 1 \\
\hline
\end{tabular}

${ }^{1}$ For example, a cell with 1,3 implies an AR model like $y_{t}=\gamma_{1} y_{t-1}+\gamma_{3} y_{t-3}+\varepsilon_{t}$.

Table 5

Parameter estimates and rejection frequencies of different AR(1) and PAR(1) models for the adjusted $\tilde{y}_{t}$ series when a PAR(1) model is the DGP (1000 replications, 100 effective observations)

\begin{tabular}{|c|c|c|c|c|c|c|}
\hline & \multicolumn{4}{|c|}{ Periodically stationary DGP } & \multicolumn{2}{|c|}{ Periodically integrated DGP } \\
\hline & $\mathrm{i}$ & ii & iii & iv & $v$ & vi \\
\hline & \multicolumn{6}{|c|}{ Nonperiodic AR(1) models } \\
\hline & 0.856 & 0.873 & 0.907 & 0.937 & 0.983 & 0.989 \\
\hline & \multicolumn{6}{|c|}{ Periodic AR(1) models } \\
\hline$\hat{\phi}_{1}$ & 0.566 & 0.707 & 1.088 & 0.837 & 0.902 & 0.999 \\
\hline$\hat{\phi}_{2}$ & 1.013 & 0.843 & 0.593 & 0.941 & 0.982 & 0.979 \\
\hline$\hat{\phi}_{3}$ & 0.872 & 0.961 & 1.053 & 1.026 & 1.031 & 0.993 \\
\hline & 1.219 & 1.061 & 1.089 & 0.965 & 1.040 & 0.985 \\
\hline \multirow[t]{2}{*}{$\hat{\phi}_{1} \hat{\phi}_{2} \hat{\phi}_{3} \hat{\phi}_{4}$} & 0.609 & 0.608 & 0.740 & 0.780 & 0.950 & 0.957 \\
\hline & \multicolumn{6}{|c|}{ Rejection frequencies of F-test for periodicity in parameters } \\
\hline$\tilde{y}_{t}$ & 99.2 & 62.7 & 99.6 & 29.2 & 41.9 & 0.0 \\
\hline
\end{tabular}

Note: The true values of $\phi_{1} \phi_{2} \phi_{3} \phi_{4}$ are given in the final column of Table 1 .

parameters of the DGPs, see the final column of Table 1. This fact implies that the outcome of the BF test for periodic integration in (9) may be robust with respect to the selected seasonal adjustment method. We could conclude that a random walk process for an adjusted series can be identified when a PIAR(1) is the DGP.

The tentatively identified autoregressive orders for univariate nonperiodic models for the $y_{t}, \Delta_{1} y_{t}$, and $\tilde{y}_{t}$ are summarized in Table 4. Practically, after the identification of an AR process, one applies diagnostic tests to check the adequacy of the identified model and its parameter estimates. Standard tests, at least based on quarterly observed variables. are the F versions of Lagrange Multiplier (LM) test 
Table 6

Estimated rejection probabilities for nonperiodic AR models corresponding to $y_{t}, \Delta_{1} y_{t}, \Delta_{4} y_{t}$ and $\tilde{y}_{t}$. The tests consider first and fourth-order residual autocorrelation, $\mathrm{LM}_{1}$ and $\mathrm{LM}_{4}$, and first order periodic autocorrelation, $\mathrm{LMP}_{1}$. The cells contain the rejection frequencies at a $5 \%$ level for 1000 replications and 100 effective observations. The DGPs are the PAR(1)'s from Table 1

\begin{tabular}{|c|c|c|c|c|c|c|c|}
\hline \multirow[b]{2}{*}{ Test } & \multirow[b]{2}{*}{ Series } & \multicolumn{4}{|c|}{ Periodically stationary DGP } & \multicolumn{2}{|c|}{ Periodically integrated DGP } \\
\hline & & $\mathrm{i}$ & ii & iii & iv & $\mathrm{v}$ & $\mathrm{vi}$ \\
\hline \multirow[t]{4}{*}{$\mathrm{LM}_{1}$} & $y_{t}$ & 13.2 & 2.4 & 1.1 & 4.9 & 19.1 & 13.3 \\
\hline & $\Delta_{1} y_{t}$ & 3.2 & 19.5 & 3.5 & 32.1 & 12.0 & 9.4 \\
\hline & $\Delta_{4} y_{1}$ & 19.5 & 22.0 & 2.5 & 13.3 & 31.4 & 10.2 \\
\hline & $\tilde{y}_{t}$ & 9.5 & 10.8 & 0.7 & 15.8 & 2.0 & 12.0 \\
\hline \multirow[t]{4}{*}{$\mathrm{LM}_{4}$} & $y_{t}$ & 31.6 & 6.6 & 6.4 & 17.2 & 22.1 & 15.2 \\
\hline & $\Delta_{1} y_{t}$ & 16.3 & 14.4 & 10.6 & 18.6 & 20.5 & 10.1 \\
\hline & $\Delta_{4} y_{1}$ & 47.8 & 52.6 & 19.4 & 51.9 & 51.4 & 57.6 \\
\hline & $\tilde{y}_{t}$ & 27.9 & 35.9 & 9.0 & 36.9 & 12.0 & 48.4 \\
\hline \multirow[t]{4}{*}{$\mathrm{LMP}_{1}$} & $y_{t}$ & 99.8 & 67.6 & 100.0 & 36.3 & 99.8 & 14.1 \\
\hline & $\Delta_{1} y_{t}$ & 99.0 & 64.9 & 100.0 & 31.2 & 99.5 & 13.2 \\
\hline & $\Delta_{4} y_{t}$ & 99.2 & 65.0 & 100.0 & 34.5 & 99.7 & 12.7 \\
\hline & $\tilde{y}_{t}$ & 99.3 & 55.4 & 99.3 & 25.5 & 99.0 & 12.0 \\
\hline
\end{tabular}

Note: The models for $y_{t}$ and $\Delta_{1} y_{t}$ include a constant and seasonal dummies, while those for $\Delta_{4} y_{t}$ and $\tilde{y}_{t}$ only contain a constant.

for residual autocorrelation of order 1 and 4 . The test statistics will be denoted as $\mathrm{LM}_{1}$ and $\mathrm{LM}_{4}$. For an $\mathrm{AR}(k)$ model, $\mathrm{LM}_{1} \sim \mathrm{F}(1, n-k-1)$ and $\mathrm{LM}_{4} \sim \mathrm{F}(4, n-k-4)$, respectively. On can also apply an LM test for a first-order periodic autocorrelation in the estimated residuals, which is proposed in (Franses, 1993). This $\mathrm{LMP}_{1}$ test is similar to the $\mathrm{LM}_{1}$ test, when the lagged residuals are multiplied with a seasonal dummy variable. An $\mathrm{F}(4, n-k-4)$-test can be derived for periodic autocorrelation of order 1 in a straightforward way. Alternatively, one may also want to use the recent tests developed by Anderson and Vecchia (1993) and Vecchia and Ballerni (1991).

The results of applying several diagnostic tests to the residuals of the AR models from Table 4 are displayed in Table 6 . The rejection frequencies for the $\mathrm{LM}_{1}$ test only twice exceed a value of $25 \%$ and those for the $\mathrm{LM}_{4}$ test are all below $60 \%$. For some of the DGPs, the rejection frequencies are close to the nominal size. Especially for the $y_{t}$ and $\Delta_{1} y_{t}$ series, it will be difficult to reject a nonperiodic AR model in case a PAR(1) process is the DGP. For the $\Delta_{4} y_{t}$ series, one may sometimes detect misspecification using the $\mathrm{LM}_{4}$ test. Further, notice that the $\mathrm{LM}_{4}$ test rejects no residual autocorrelation for the $\tilde{y}_{t}$ series in several cases. This means that one has to include $\tilde{y}_{t-4}$ in a model for $\tilde{y}_{t}$, which is quite unusual for a seasonally adjusted series. However, the $\mathrm{LMP}_{1}$ test seems quite powerful in rejecting the null hypothesis of no periodicity in the estimated residuals. Hence, any underlying periodicity can 
Table 7

Estimated acceptance and rejection probabilities based on the BF-test for testing periodic integration. The null hypothesis is that $\phi_{1} \phi_{2} \phi_{3} \phi_{4}=1$ in the model $x_{t}=\phi_{s} x_{t-1}+\varepsilon_{t}$, where $x_{t}$ is the original series $y_{t}$ or the X-11 adjusted series $\tilde{y}_{t}$. The cells contain the rejection frequencies for 1000 replications with 100 effective observations. The columns (i)-(iv) refer to the power, while the columns (v) and (vi) refer to the empirical size. The DGPs are the PAR(1) processes from Table 1

\begin{tabular}{|c|c|c|c|c|c|c|c|}
\hline \multirow[b]{2}{*}{ Series } & \multirow[b]{2}{*}{$\begin{array}{l}\text { Nominal } \\
\text { size }\end{array}$} & \multicolumn{4}{|c|}{ Periodically stationary DGP } & \multicolumn{2}{|c|}{ Periodically integrated DGP } \\
\hline & & $\mathrm{i}$ & ii & iii & iv & $\mathrm{v}$ & vi \\
\hline \multirow[t]{2}{*}{$y_{t}$} & $5 \%$ & 86.5 & 91.7 & 48.1 & 38.4 & 4.7 & 4.3 \\
\hline & $10 \%$ & 97.7 & 97.9 & 70.9 & 63.3 & 9.5 & 9.3 \\
\hline \multirow[t]{2}{*}{$\tilde{y}_{t}$} & $5 \%$ & 86.0 & 84.6 & 56.2 & 30.6 & 4.8 & 3.3 \\
\hline & $10 \%$ & 97.1 & 96.6 & 78.4 & 53.6 & 10.0 & 7.9 \\
\hline
\end{tabular}

be detected for the $\tilde{y}_{t}$ series by applying a test for periodic parameters or for periodic error processes, see Table 5.

Considering Table 5, one notes that the $\prod_{i=1}^{4} \hat{\phi}_{i}$ in a PAR(1) model obtains a value close to the parameters in the DGPs. This suggests that linear filtering may not effect the outcome of the BF test for periodic integration in (9). This conjecture is emphasized by the results in Table 7. The DGPs (v) and (vi) are periodically integrated implying that the estimated rejection probabilities correspond to the nominal significance level $1-\alpha=0.95$. From the last two columns, one can observe that this is the case for $y_{t}$ as well as for $\tilde{y}_{t}$. The other columns in Table 7 display the power of the test statistic. It is clear that the power may not be high at a $5 \%$ level, but that the power for the $y_{t}$ and $\tilde{y}_{t}$ series is almost equal.

Our Monte-Carlo experiments on the analysis of the effects of a misspecification can be summarized as follows. When a PAR model is the DGP, one should identify reasonably low-order nonperiodic autoregressive models for the raw series and AR(1) models for the seasonally adjusted series $\tilde{y}_{t}$. Using standard diagnostic tests for detecting residual autocorrelation, one cannot reject the nonperiodic models. As expected, a test for periodicity in the residuals yields the best results. According to Boswijk and Franses (1992), a sensible strategy therefore seems to be to begin with a univariate analysis by identifying a PAR process, estimating its parameters, and to test if the autoregressive parameters are periodically varying or not. If not, one can apply a linear correction technique. Remembering the role of the adjustment filter in (16), it can be concluded that the performance of the BF test for periodic integration is only weakly affected. Hence, the same test may also be applied to seasonally adjusted data. In the next section, the real GNP series for Germany will be analyzed keeping the results of the above Monte-Carlo study in mind. 


\section{An empirical example}

Consider again the log of the real GNP series for Germany, 1960.1-1990.4, to be denoted by $y_{t}$. The (partial) autocorrelations of $y_{t}$ and its transformed versions $\Delta_{1} y_{t}, \Delta_{4} y_{t}$ and $\Delta_{1} \Delta_{4} y_{t}$ are displayed in Table 8. MicroTSP version 7.0 is used for the computations. The estimated autocorrelations of $y_{t}$ and $\Delta_{4} y_{t}$ mimic the behaviour of the autocorrelation function corresponding to the DGPs (v) and (vi) in Table 2. Based on these (partial) autocorrelations, nonperiodic AR models can be easily identified. The model orders for the four series, i.e., $y_{t}, \Delta_{1} y_{t}, \Delta_{4} y_{t}$ and $\Delta_{1} \Delta_{4} y_{t}$, are reported in the first row of Table 9. For the sake of completeness, the parameters of the four identified AR models are estimated, and some diagnostic results are displayed in Table 9. It can be observed that the LM tests for nonperiodic residual autocorrelation and for autoregressive conditional heteroskedastic (ARCH) patterns usually do not indicate a severe misspecification. Furthermore, the normality of the estimated residuals cannot be rejected using a $\chi^{2}(2)$-test. However, the $\mathrm{LMP}_{1}$ test for periodicity in the residuals suggests that the corresponding null hypothesis is rejected at a $5 \%$ level. It should be mentioned that an application of the HEGY test [see (Hylleberg et al., 1990)] yields that the $\Delta_{4}$ filter is a "good" choice for this time series, see (Wolters, 1992). An appropriate model for $\Delta_{4} y_{t}$ turns out to be

$$
\begin{aligned}
\Delta_{4} y_{t}= & 0.0078+0.743 \Delta_{4} y_{t-1}+\varepsilon_{t}, \\
& (0.0024) \quad(0.060),
\end{aligned}
$$

(with estimated standard errors in parentheses). The Dickey-Fuller test applied to (17) yields a value of -4.269 , and hence the use of the $\Delta_{1} \Delta_{4}$ filter implies overdifferencing. Anyway, given the results of the $\mathrm{LMP}_{1}$ test for the nonperiodic AR models listed in Table 9, we conclude that a periodic model gives a useful description of the German GNP series.

As already noted in Section 3, a favourable procedure of modelling is to start with the specification of a general PAR process, and to test whether the autoregressive parameters are indeed periodically varying. The order of the initial PAR model can be estimated and can be tested using $\mathrm{F}$-test for the joint hypothesis $\mathbf{H}_{0}$ : $\left(\phi_{p s}\right)_{s=1,2,3,4} \neq 0$. For the German data, the F-test for the significance of the $\phi_{2 s}$ in a PAR(2) model obtains a value of 8.516 , while F-tests for the significance of $\phi_{3 s}$ and $\phi_{4 s}$ in PAR(3) and PAR(4) models, respectively, yield values which are not significant even at a $20 \%$ level. An $F(6,110)$-test for the periodicity of the AR parameters in this PAR(2) process results in $\hat{F}=43.485$. Hence, we conclude that a PAR(2) process like (10) is appropriate for modelling the $y_{t}$ series, i.e.,

$$
A_{0} Y_{T}=A_{1} Y_{T-1}+\mu+\omega_{T},
$$


Table 8

Estimated (partial) autocorrelations of the log of seasonally unadjusted real German GNP series for different kinds of differencing

\begin{tabular}{|c|c|c|c|c|c|c|c|c|}
\hline \multicolumn{9}{|c|}{ Degree of differencing } \\
\hline \multirow[t]{2}{*}{ Lag } & \multicolumn{2}{|c|}{$y_{t}$} & \multicolumn{2}{|l|}{$\Delta_{1} y_{1}$} & \multicolumn{2}{|l|}{$\Delta_{4} y_{t}$} & \multicolumn{2}{|l|}{$\Delta_{1} \Delta_{4} y_{t}$} \\
\hline & $\mathrm{ACF}$ & PACF & $\mathrm{ACF}$ & PACF & $\mathrm{ACF}$ & PACF & $\mathrm{ACF}$ & PACF \\
\hline 1 & 0.948 & 0.948 & -0.150 & -0.150 & 0.740 & 0.740 & -0.056 & -0.056 \\
\hline 2 & 0.904 & 0.056 & -0.468 & -0.502 & 0.523 & -0.055 & -0.088 & -0.091 \\
\hline 3 & 0.895 & 0.329 & -0.079 & -0.355 & 0.367 & -0.002 & 0.084 & 0.074 \\
\hline 4 & 0.889 & 0.089 & 0.710 & 0.552 & 0.167 & -0.200 & -0.198 & -0.201 \\
\hline 5 & 0.841 & -0.321 & -0.152 & -0.010 & 0.065 & 0.059 & -0.142 & -0.156 \\
\hline 6 & 0.799 & 0.005 & -0.424 & -0.053 & 0.018 & 0.014 & 0.053 & -0.006 \\
\hline 7 & 0.790 & 0.126 & -0.052 & -0.109 & -0.060 & -0.096 & 0.054 & 0.062 \\
\hline 8 & 0.783 & 0.073 & 0.540 & -0.017 & -0.158 & -0.166 & -0.247 & -0.279 \\
\hline 9 & 0.736 & -0.208 & -0.084 & 0.079 & -0.098 & 0.245 & -0.005 & -0.106 \\
\hline 10 & 0.697 & 0.011 & -0.402 & -0.076 & -0.057 & -0.008 & 0.058 & -0.011 \\
\hline 11 & 0.689 & 0.087 & -0.062 & -0.111 & -0.055 & -0.053 & -0.159 & -0.132 \\
\hline 12 & 0.685 & 0.064 & 0.490 & 0.074 & 0.017 & 0.046 & 0.023 & -0.119 \\
\hline SE & \multicolumn{2}{|c|}{0.090} & \multicolumn{2}{|c|}{0.090} & \multicolumn{2}{|c|}{0.091} & \multicolumn{2}{|c|}{0.092} \\
\hline
\end{tabular}

Note: The $y_{t}$ series is the log of the seasonally unadjusted real German GNP series for 1960.1-1990.4. The column for $\Delta_{1} y_{t}$ concerns the residuals of the regression of $\Delta_{1} y_{t}$ on four seasonal dummies.

Table 9

Diagnostics for nonperiodic AR models for the unadjusted real German GNP series

\begin{tabular}{|c|c|c|c|c|}
\hline \multirow[b]{2}{*}{ Model order } & \multicolumn{4}{|c|}{ Degree of differencing } \\
\hline & $\begin{array}{l}y_{t} \\
1,4,5\end{array}$ & $\begin{array}{l}\Delta_{1} y_{t} \\
1,2,3,4\end{array}$ & $\begin{array}{l}\Delta_{4} y_{t} \\
1\end{array}$ & $\begin{array}{l}\Delta_{1} \Delta_{4} y_{t} \\
0\end{array}$ \\
\hline \multicolumn{5}{|l|}{ Diagnostics $^{a}$} \\
\hline $\mathrm{LM}_{1}$ & 0.837 & 0.954 & 0.818 & 0.365 \\
\hline $\mathrm{LM}_{4}$ & 2.202 & 1.424 & 1.909 & 1.737 \\
\hline $\mathrm{LMA}_{1}$ & 2.531 & 1.460 & 0.064 & 2.952 \\
\hline $\mathrm{LMA}_{4}$ & 1.594 & 2.283 & $3.559^{b}$ & 2.982 \\
\hline$\chi^{2}(2)$ & 5.778 & 1.070 & 3.711 & 3.061 \\
\hline $\mathrm{LMP}_{1}$ & $4.883^{b}$ & $4.344^{b}$ & $4.525^{b}$ & $5.748^{b}$ \\
\hline
\end{tabular}

${ }^{a}$ The models for $y_{t}$ and $\Delta_{1} y_{t}$ include four seasonal dummies, the other two models include a constant. All models are estimated using 119 observations.

${ }^{b}$ Significant at a $5 \%$ level

Note: The definition of the diagnostics is: $\mathbf{L M}_{i}$ is the $\mathbf{F}(i, n-k-i)$-test statistic for the null hypothesis of no residual autocorrelation of order $i$ (where $k$ is the number of regressors), LMA $_{i}$ is a similar test statistic for $i$ th order ARCH effects, $\chi^{2}(2)$ denotes the test statistic of a normality test based on the estimated residuals, $\mathrm{LMP}_{1}$ is an $\mathrm{F}(4, n-k-4)$-test statistic to detect periodicity in the first order lagged residuals. 
with

$$
A_{0}-A_{1} z=\left[\begin{array}{cccc}
1 & 0 & +0.375 z & -1.360 z \\
-0.279 & 1 & 0.214) & (0.192) \\
(0.150) & & & -0.684 z \\
0.320 & -1.237 & 1 & (0.153) \\
(0.146) & (0.154) & & 0 \\
0 & -0.421 & -0.638 & 1
\end{array}\right], \quad \mu=\left[\begin{array}{c}
0.017 \\
(0.135) \\
0.201 \\
(0.064) \\
0.514 \\
(0.072) \\
0.310 \\
(0.127)
\end{array}\right] .
$$

The $\mathrm{LM}_{1}$ and $\mathrm{LM}_{4}$ test statistics for (18) are $\mathrm{LM}_{1}=0.929$ and $\mathrm{LM}_{4}=1.687$, while the $\mathrm{LM}$ tests for the ARCH effects of order 1 and 4, i.e., $\mathrm{LMA}_{1}$ and $\mathrm{LMA}_{4}$, are $\mathrm{LMA}_{1}=0.106$ and $\mathrm{LMA}_{4}=1.419$, the $\chi^{2}(2)$ normality test statistic obtains a value of 3.823 and the $\mathrm{LMP}_{1}=0.467$. A regression of the squared estimated residuals on a constant and three seasonal dummies yields an $\hat{\mathrm{F}}=2.779$, which is significant at a $5 \%$ level, indicating that there may be some seasonal heteroskedasticity. However, we observed that a few observations before 1963 caused this F-test to be significant. Hence, it is decided not to extend the model in (18) with an $\varepsilon_{s t}$ error process. In summary, the model (18) seems adequately specified.

As can be expected for a time series with a stochastic trend, the correlations between the parameter estimates in (18) are very high. This effect is caused by the $y_{t}$ to be likely nonstationary. This series needs to be differenced for any further analysis. However, the estimation results in (18) are useful to support the hypothesis of the presence of unit roots in $Y_{T}$. Indeed, the characteristic equation of (18) is

$$
\left|A_{0}-A_{1} z\right|=1-1.0003 z+0.0345 z^{2}=0,
$$

with the solutions $z_{1}=0.965$ and $z_{2}=0.036$. The BF test in (9) for periodic integration gives $\mathrm{BF}=-2.213$. Hence, the null hypothesis that one of these solutions is equal to 1 cannot be rejected. In turn this implies that there are three cointegration relations between the elements of $Y_{T}$. Model (18) may now be rewritten in the differencing form (13). The corresponding parameters are as follows.

$$
\begin{array}{cccc}
\hat{\alpha}_{1}=1.025, & \hat{\alpha}_{2}=0.962, & \hat{\alpha}_{3}=0.912, & \hat{\alpha}_{4}=1.113, \\
(0.011) & (0.011) & (0.009) & (0.012) \\
\hat{\beta}_{1}=\begin{array}{c}
0.338, \\
(0.195)
\end{array} & \hat{\beta}_{2}=-0.675, & \hat{\beta}_{3}=0.351, & \hat{\beta}_{4}=-0.424, \\
(0.152) & (0.154) & (0.186)
\end{array}
$$

and the differencing filter is $\left(1-\hat{\alpha}_{s} B\right)$. An $F(3,111)$-test for the hypothesis that $\alpha_{s}=\alpha=1$ has a value of 20.841 , and the F-test for the hypothesis that $\beta_{s}=\beta$ in a PAR(1) model for $\left(1-\hat{\alpha}_{s} B\right) y_{t}$ yields $\hat{F}=10.662$. Of course, one may want to further simplify the PAR(2) model, but this is not pursued here. 


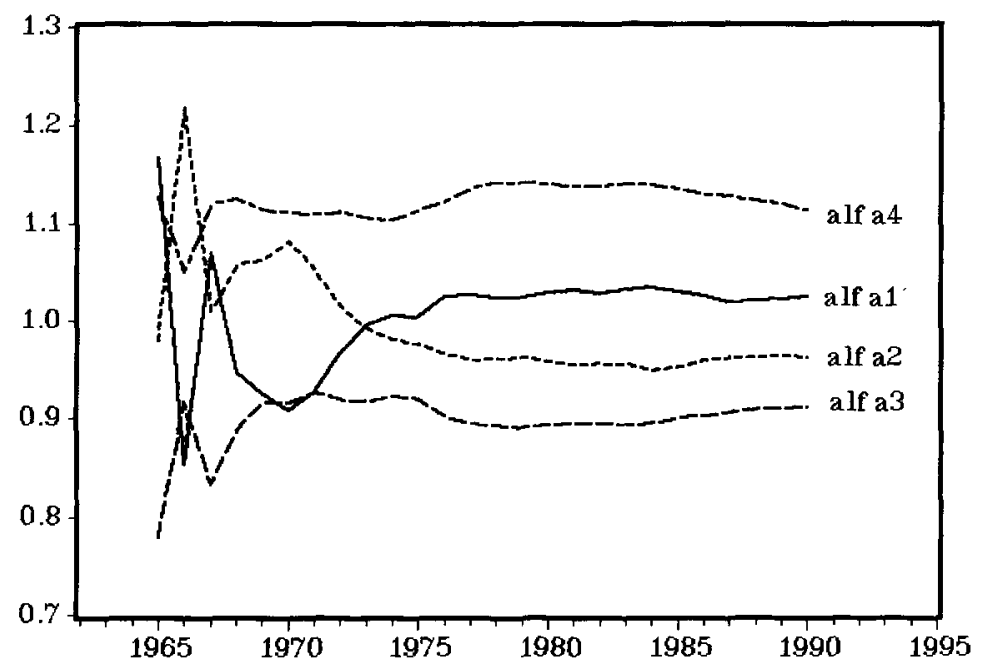

Fig. 5. Vertical axis: the recursive non-linear least squares estimates of $\alpha_{s}, s=1,2,3,4$, in the differencing filter $1-\alpha_{s} B$. Horizontal axis: the time in years.

To test whether a periodic autoregressive model with a single unit root is a model with constant parameters or not, consider the recursively estimated values of the $\alpha_{s}$ parameters under the restriction that $\alpha_{1} \alpha_{2} \alpha_{3} \alpha_{4}=1$ as they are given in Fig. 5. The estimates for $\alpha_{s}(s=1,2,3,4)$ seem fairly constant over time indeed. The $\mathrm{F}(3, m)$-test for the hypothesis $\mathrm{H}_{0}: \alpha_{s}=1$ yields a sequence of values, of which $\hat{\mathrm{F}} \geqslant 5.873$, when the sample runs from 60.3 through 68.4 , and of $\hat{F} \leqslant 22.870$ when the sample ends in 1989.4. These outcomes emphasize the robustness of the PAR(2) model with a single unit root and of the inappropriateness of the $(1-B)$ filter throughout the sample. Fig. 6 displays the $y_{t}-\hat{\alpha}_{s} y_{t-1}$ series in the quarters 2, 3 and 4. Comparing these with the graphs in Fig. 4 implies that only the periodic differencing filter seems to yield a residual process which does not contain a stochastic trend anymore.

To apply the seasonal adjustment filter in (16), it is a standard practice to forecast the $y_{t}$ series using a model like (17), and to use these forecasts to obtain current values of a seasonally corrected series. However, since the model in (17) appears to be misspecified, only the in-sample observations are used to construct the $\tilde{y}_{t}$ series. This $\tilde{y}_{t}$ series now covers the period 1967.1 to 1983.4, i.e., it has 68 realizations. The (unreported) (partial) autocorrelations of the $\tilde{y}_{t}$ and the $\Delta_{1} \tilde{y}_{t}$ series are similar to those in Table 2. The $\tilde{y}_{t}$ series seems to be integrated of order one, a conjecture which is confirmed by the Dickey-Fuller test value of -1.641 , which is obtained from a regression model with a constant and no lags. An adequate model for $\Delta_{1} \tilde{y}_{t}$ therefore seems to be

$$
\Delta_{1} \tilde{y}_{t}=\underset{(0.0014)}{0.0072}+v_{t} .
$$

We have $\mathrm{LM}_{1}=0.257 . \quad \mathrm{LM}_{4}=1,468, \quad \mathrm{LMA}_{1}=0.177, \quad \mathrm{LMA}_{4}=0.410$, $\chi^{2}(2)=0.024$, and $\mathrm{LMP}_{1}=1.433$. The $\mathrm{F}(3,59)$-test for periodic autoregressive 


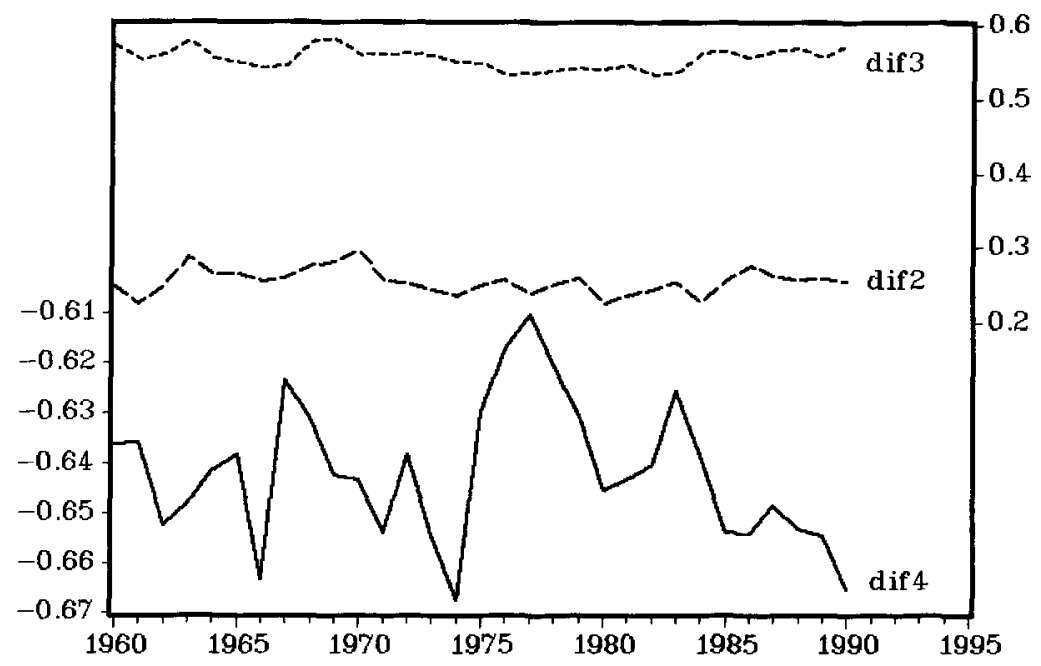

Fig. 6. Vertical axis: the periodically differenced time series $\left(1-\alpha_{s} B\right) y_{t}$ in quarter 2,3 and 4 . Horizontal axis: the time in years.

parameters when a PAR( 1$)$ is fitted to $\tilde{y}_{t}$ gives $\hat{\mathrm{F}}=0.858$, and hence the periodicity seems to be removed by applying a linear seasonal adjustment filter. To verify whether this is due to a subsample, a PAR(2) model as in (18) is estimated and the corresponding $F(6,56)$-test for periodic parameters yields $\hat{F}=20.157$. Hence, this GNP series is one of the cases in which the underlying periodicity cannot be detected after a linear Census $X-11$ filtering. The model for $\tilde{y}_{t}$ is a random walk, and consequently one may question its relevance to forecasting and considering turning points. The PAR(2) model with a single unit root, however, relates trends to seasonality, and hence it pays off to model seasonality together with the underlying trend and any cyclical behavior. This is a very important aspect of the model in (18) with an imposed unit root, i.e., seasonality, cycles and trend are modeled simultaneously. The pattern of the multi-step forecasts from (18) with an imposed unit root further allows that seasonality can change as in Fig. 2, while forecasts from (17) will show the well-known constant pattern, which is dependent on the most recent observations.

\section{Discussion}

In this paper the effects of specific seasonal adjustment procedures on a periodic autoregressive time series are studied. Making a unit root approach, such processes are well-suited for modeling seasonal time series. Such series display slowly changing seasonal fluctuations dominated by a stochastic trend. A piece of evidence is the German real GNP series, which, consequently, is used as a running example throughout this paper. 
Traditional methods to analyze quarterly time series assume that the transformation of the series to annual growth rates is appropriate, or that a linear Census X-11 filter can be used. A Monte-Carlo study shows that seasonal adjustment does not entirely remove the intrinsic periodicity, that a test for periodic integration is not affected, and that the most sensible practical strategy seems to be to start with a general periodic autoregressive model. If the nonperiodicity of the autoregressive parameters will not be removed then seasonal adjustment procedures can be applied.

Keeping these results in mind, further research should investigate more seasonally adjusted series, and test if the series are periodically integrated. In (Franses, 1992b) further evidence is already given. A natural extension of the concept of periodic integration seems to be the concept of periodic cointegration, see (Birchenhall et al., 1989) and (Franses and Kloek, 1991). Applications of these concepts are usually considered for nonadjusted time series. Whether linear seasonal adjustment effects detecting a periodic cointegration relationship is yet to be studied.

\section{Acknowledgements}

The financial support from the Royal Netherlands Academy of Arts and Sciences is gratefully acknowledged. The extensive comments from two anonymous referees and from participants of the International Symposium on Forecasting in Pittsburgh (1993) are appreciated. Thanks are also due to Jürgen Wolters for giving access to the German data, and to Richard Paap and Paula Pilaar for their programming efforts.

\section{References}

Anderson, P.L. and A.V. Vecchia, Asymptotic results for periodic auto-regressive moving-average processes, J. Time Ser. Anal., 14 (1993) 1-18.

Barsky, R.B. and J.A. Miron, The seasonal cycle and the business cycle, J. Political Economy, 97 (1989) 503-534.

Beaulieu, J.J., J.K. MacKie-Mason and J.A. Miron, Why do countries and industries with large seasonal cycles also have large business cycles?, Quart. J. Econom., 107 (1992) 621-656.

Birchenhall, C.R., R.C. Bladen-Hovell, A.P.L. Chui, D.R. Osborn and J.P. Smith, A seasonal model of consumption, Econom, J., 99 (1989) 837-43.

Boswijk, H.P. and P.H. Franses, Testing for periodic integration, Econometric Institute Report 9216 (Erasmus University, Rotterdam, 1992) Submitted.

Canova, F. and E. Ghysels, Changes in seasonal patterns: are they cyclical?, J. Econom. Dynamics Control (forthcoming).

Engle, R.F. and B.S. Yoo, Forecasting and testing in cointegrated systems, J. Econometrics, 35 (1987) 143-159.

Franses, P.H., A multivariate approach to modeling univariate seasonal times series, J. Econometrics. to appear.

Franses, P.H., Seasonality in consumer confidence in some European countries, Econometric Institute Report 9261 (1992a). 
Franses, P.H., A stylized fact re-analyzed, Econometric Institute Report 9275 (Erasmus University, Rotterdam, 1992b).

Franses, P.H., Periodically integrated subset autoregressions for Dutch industrial production and money stock, Journal of Forecasting, 12 (1993) 601-613.

Franses, P.H. and T. Kloek, A periodic cointegration model for quarterly consumption, Econometric Institute Report 9172, (Erasmus University, Rotterdam, 1991).

Franses, P.H. and G. Romijn, Periodic integration in UK macroeconomic time series, Internat. J. Forecasting, to appear.

Fuller, W.A. Introduction to statistical time series (Wiley, New York, 1976).

Ghysels, E., Are business cycle turning points uniformly distributed throughout the year?, Discussion paper 3891 (CRDE, Montreal, 1991).

Ghysels, E. and A. Hall, Testing periodicity in some linear macroeconomic models, CRDE Montreal Unpublished Manuscript (1992).

Hylleberg, S., R.F. Engle, C.W.J. Granger and B.S. Yoo, Seasonal Integration and Cointegration, J. Econometrics, 44 (1990) 215-238.

Laroque, G., Analyse d'une methode de desaisonnalisation: le programme X11 du US Bureau of Census, version trimestrielle, Annales de l'INSEE, 28, (1977) 105-127.

Osborn, D.R., Seasonality and Habit Persistence in a Life-Cycle Model of Consumption, J. Appl. Econometrics, 3 (1988) 255-266.

Osborn, D.R., The Implications of Periodically Varying Coefficients for Seasonal Time-Series Processes, J. Econometrics. 28 (1991) 373-384.

Osborn, D.R. and J.P. Smith, The Performance of Periodic Autoregressive Models in Forecasting Seasonal U.K. Consumption, J. Business Econom. Statistics, 7 (1989) 117-127.

Tiao, G.C. and M.R. Grupe, Hidden periodic autoregressive moving average models in time series data, Biometrika, 67 (1988) 365-373.

Vecchia, A.V. and R. Ballerini, Testing for periodic autocorrelations in seasonal time series data, Biometrika, 78 (1991) 53-63.

Wolters, J., Persistence and seasonality in output and employment of the Federal Republic of Germany, Recherches Economiques de Louvain, 58 (1992) 421-439. 